Review

\title{
Biogenesis and Dynamics of the Coronavirus Replicative Structures
}

\author{
Marne C. Hagemeijer ${ }^{\dagger}$, Peter J.M. Rottier and Cornelis A.M. de Haan * \\ Virology Division, Department of Infectious Diseases \& Immunology, Faculty of Veterinary \\ Medicine, Utrecht University, Yalelaan 1, 3584 CL Utrecht, The Netherlands; E-Mails: \\ mch143@andromeda.rutgers.edu (M.C.H.); p.rottier@uu.nl (P.J.M.R.) \\ $\dagger$ Current address: Host-Pathogen Dynamics Group, Federated Department of Biological Sciences, \\ Rutgers University, Newark, NJ 07102, USA. \\ * Author to whom correspondence should be addressed; E-Mail: c.a.m.dehaan@uu.nl; \\ Tel.: +31-30-253-4195; Fax: +31-30-253-6723.
}

Received: 21 October 2012; in revised form: 14 November 2012 / Accepted: 15 November 2012 / Published: 21 November 2012

\begin{abstract}
Coronaviruses are positive-strand RNA viruses that are important infectious agents of both animals and humans. A common feature among positive-strand RNA viruses is their assembly of replication-transcription complexes in association with cytoplasmic membranes. Upon infection, coronaviruses extensively rearrange cellular membranes into organelle-like replicative structures that consist of double-membrane vesicles and convoluted membranes to which the nonstructural proteins involved in RNA synthesis localize. Double-stranded RNA, presumably functioning as replicative intermediate during viral RNA synthesis, has been detected at the double-membrane vesicle interior. Recent studies have provided new insights into the assembly and functioning of the coronavirus replicative structures. This review will summarize the current knowledge on the biogenesis of the replicative structures, the membrane anchoring of the replication-transcription complexes, and the location of viral RNA synthesis, with particular focus on the dynamics of the coronavirus replicative structures and individual replication-associated proteins.
\end{abstract}

Keywords: coronavirus; replication complex; nonstructural protein; membrane rearrangements; protein-protein interactions; live cell imaging; dynamics; RNA synthesis 


\section{Introduction}

Positive-strand RNA (+RNA) viruses are the most abundant viruses in nature. Many important pathogens belong to this category, including poliovirus $(\mathrm{PV})$, hepatitis $\mathrm{C}$ virus $(\mathrm{HCV})$ and the severe acute respiratory syndrome (SARS)-coronavirus $(\mathrm{CoV})$. A distinctive common feature of $+\mathrm{RNA}$ viruses is the replication of their genomes in the cytoplasm of the host cell in association with rearranged cellular membranes that are remodeled into organelle-like membranous structures to which the viral replication-transcription complexes (RTCs) localize.

The various membrane rearrangements observed in +RNA virus-infected cells range in size from 40 to $400 \mathrm{~nm}$, contain lipids that are derived from various cellular compartments and demonstrate an impressively diverse plethora of morphologies that include, among others, clusters of vesicles for the Picorna- and Togaviridae, spherule-like invaginations for the Bromoviridae and Nodaviridae, and vesicle packets and membranous webs for the Flaviviridae (reviewed in [1-3]). These membrane rearrangements seem to be beneficial for (i) sequestering and concentrating all viral and cellular components necessary for viral RNA synthesis and (ii) to provide a protective microenvironment against virus-elicited host defense mechanisms.

Also coronaviruses (CoVs), enveloped +RNA viruses that belong to the family Coronaviridae, extensively rearrange cellular membranes into organelle-like replicative structures during infection. These replicative structures consist of double membrane vesicles (DMVs) and convoluted membranes (CMs). The viral replicase proteins involved in RNA synthesis localize to both these structures, while double-stranded RNA (dsRNA), presumably functioning as replicative intermediate during viral RNA synthesis, has been detected at the DMV interior [4,5]. Recent studies have provided new insights into the assembly and functioning of the $\mathrm{CoV}$ replicative structures. This review will summarize the current knowledge on the biogenesis of the replicative structures, the membrane anchoring of the RTCs, and the location of viral RNA synthesis, with particular focus on the dynamics of the coronavirus replicative structures and individual replication-associated proteins.

\section{Coronavirus Genome Organization and Gene Expression}

The Coronaviridae are a family of evolutionary related, enveloped +RNA viruses that together with the Arteriviridae and the Roniviridae, belong to the order of the Nidovirales. Historically, CoVs have been recognized as important infectious agents for domestic livestock, poultry and companion animals. In contrast to the animal viruses, human $\mathrm{CoVs}(\mathrm{HCoVs})$ have been associated with relatively mild upper and lower respiratory tract infections, including ordinary common colds. However, in 2002-2003 the outbreak of a novel $\mathrm{HCoV}$ in China, causing severe fatal atypical pneumonia in infected individuals, demonstrated that $\mathrm{HCoVs}$ are also able to induce severe life-threatening disease in humans. This virus was called the SARS-CoV [6,7] and emerged in the human population from an animal reservoir, probably originating from bats, with palm civet cats acting as intermediate hosts [8,9].

Among the +RNA viruses, CoVs clearly distinguish themselves by carrying the most complex and largest genomes, which range in size from $\sim 26$ to $32 \mathrm{~kb}$ [10]. Despite the variation in size, the overall genome organization of the various CoVs is quite conserved. The genome contains all the genetic information necessary to direct both the synthesis of full-length genomic RNA (replication) and the 
(discontinuous) production of subgenomic mRNAs (transcription) [11]. The linear +RNA genome of CoVs is 3' polyadenylated and has a 5' cap structure, thereby mimicking cellular mRNAs. The 5' and 3' ends of the genome contain untranslated regions (UTRs) with cis-acting elements that are important for replication and transcription. Two-thirds of the genome consists of two large open reading frames (ORFs), ORF1a and ORF1b. The remaining 3' one-third part encodes the structural proteins interspersed with sequences encoding some accessory proteins. A schematic picture of the prototype mouse hepatitis virus (MHV) genome is shown in Figure 1A.

The structural and the accessory proteins are expressed from a nested set of 3' coterminal subgenomic ( $\mathrm{sg}$ ) mRNAs that are generated via discontinuous transcription during subgenome-length minus-strand RNA synthesis [12,13]. The RNA-dependent RNA polymerase (RdRp) copies the genomic positive-sense RNA into a negative-sense template until it reaches a transcription-regulation sequence (TRS). At this point, RNA synthesis may either continue or the RdRp may relocate to the $5^{\prime}$ end of the genome and complete the negative-sense sgRNA. These negative-sense sgRNAs serve as templates for the synthesis of the corresponding positive-sense sgRNAs. As a result, the positive-sense sgRNAs form a nested set of mRNAs, which extend for different lengths from a common 3 ' terminus while also having a common $5^{\prime}$ end, which is known as the leader sequence. Generally, only the $5^{\prime}$ first unique gene of each sgRNA is translated (reviewed in [11]). Minus- and plus-strand RNA synthesis is already detected at 75 to $90 \mathrm{~min}$ post infection (p.i.) [11]. Minus-strand synthesis, of which the resulting RNA species are mainly present as double-stranded intermediates because of their association with plus-strand RNA molecules, peaks at 5 to $6 \mathrm{~h}$ p.i. after which the synthesis declines but does not stop [14]. The plus-stranded RNAs are produced in a 50- to 100-fold excess over their minus-strand counterparts $[11,15]$.

The viral replicase is encoded by the two most 5' ORFs of the genomic RNA, ORF1a and ORF1b. Translation of ORF1a and ORF1b of the genomic RNA generates two very large replicase polyproteins (pp), ppla and pplab. The latter is synthesized via a -1 ribosomal frameshift mechanism mediated by a pseudoknot structural element at the end of ORF1a [16,17]. These replicase polyproteins are extensively processed by viral proteinases (reviewed in [18]), resulting in the generation of sixteen nonstructural proteins (nsps). A schematic representation of the CoV replicase polyprotein is shown in Figure 1B.

\section{Coronavirus Nonstructural Proteins}

\subsection{Viral Proteinases}

The CoV nsps form together with the nucleocapsid $(\mathrm{N})$ protein, and presumably several host proteins, the membrane-associated RTCs. To generate the functional $\mathrm{CoV}$ replication complexes, the replicase polyproteins ppla and pplab have to be proteolytically processed to liberate the sixteen individual protein products. Cleavage of ppla and pplab is performed by two viral proteinases that reside in nsp3 and nsp5: the papain-like proteases (PLpro1 and PLpro2) located in nsp3 and the chymotrypsin-like cysteine proteinase (3CLpro), or main protease (Mpro), present in nsp5 (reviewed in [18]). Besides the mature nsps, also the intermediate and precursor polyproteins are likely to be 
functionally important. Hence, the cleavage of these proteins may somehow be involved in the temporal regulation of plus and/or minus sense viral RNA synthesis [18-20].

Figure 1. Schematic representation of the coronavirus mouse hepatitis virus (MHV)A59 genome, replicase polyprotein organization and membrane topology. (A) Schematic representation of the +RNA genome of MHV-A59. The coronavirus genome contains a $5^{\prime}$ cap structure and a $3^{\prime}$ poly(A) tail, together with untranslated regions (UTRs). The first two-thirds of the genome consist of two large open-reading frames (ORFs), ORF1a and ORF1b, which are translated into two large replicase polyproteins (ppla and pplab). Pplab is synthesized via a -1 ribosomal frameshift mechanism at the end of ORF1a (RFS). The final one-third of the genome contains the canonical CoV structural proteins-encoding genes (S, E, M and N), interspaced by several accessory genes (2a, HE, 4, 5a); (B) A schematic representation of pplab is shown. The coronavirus polyproteins are processed by viral proteinases residing in nsp3 (PLpro1 and PLpro2; grey arrowheads indicate cleavage sites) and nsp5 (Mpro; black arrowheads indicate cleavage sites), thereby generating 16 mature nsps. Hydrophobic domains (TM1, TM2 and TM3) in nsp3, nsp4 and nsp6 are indicated, together with predicted and identified RNA(-modifying) enzymes: the RNA-dependent RNA polymerase (RdRP; nsp12), the helicase (Hel; nsp13), the exonuclease (ExoN; nsp14), the uridylate-specific endoribonuclease (N; nsp15), and the methyl transferase (MT; nsp16); (C). Schematic representation of the topology of the coronavirus polyprotein. Only the part of the polyprotein is shown that contains the hydrophobic domains (indicated by boxes) residing in nsp3, nsp4 and nsp6. Nsp3 and nsp6 contain hydrophobic domains that do not span the lipid bilayer. Mpro indicates the viral protease residing in nsp5, in between nsp4 and nsp6.

\section{A}

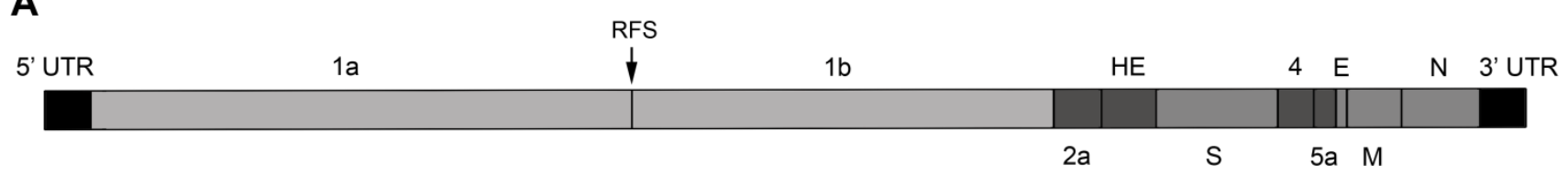

B

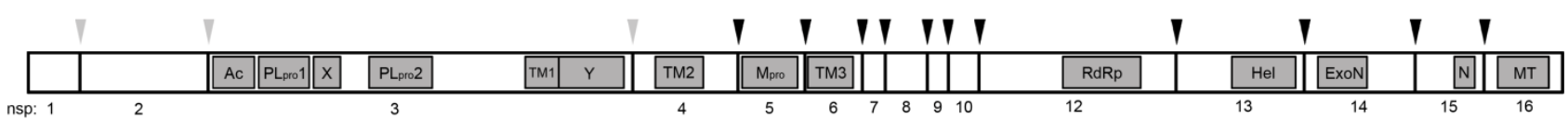

C

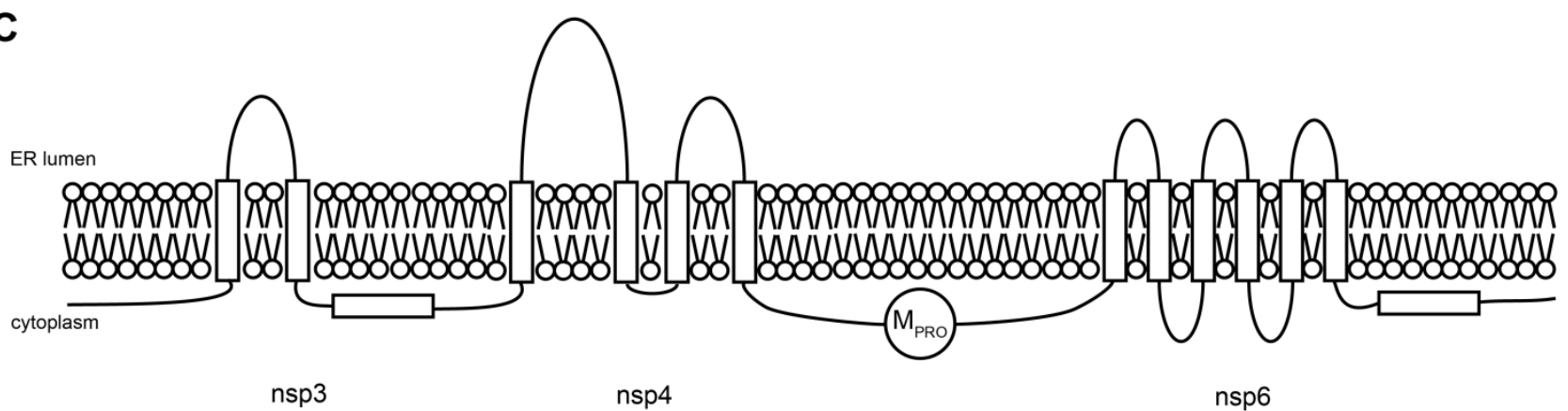




\subsection{RNA-modifying Enzymes}

The key enzyme involved in genome replication is the RNA-dependent RNA polymerase (RdRp), which is present in nsp12 [10]. The nsp12 protein is able to utilize both homo- and heteropolymeric RNAs as template but its RdRp activity is dependent on primers to copy the viral RNA [21]. These primers might be produced by a non-canonical RdRp activity that has been described for the nsp8-encoded 'RNA primase', as this protein is able to produce short oligonucleotides complementary to the RNA genome [22]. Nsp8 has been shown to associate together with nsp7 into a hexadecameric complex, consisting of eight copies of each protein, thereby forming a channel that can harbor RNA and may serve as a processivity factor for nsp12 [23]. Nsp8 also interacts with nsp7 in a 1:2 ratio [24] and is able to synthesize much longer transcripts [24,25].

The CoV nsp13 protein contains a superfamily 1 helicase domain with an amino-terminal zincbinding domain that is important for the unwinding activity of duplex RNA (and DNA) in a 5'-to-3' direction [26-28]. The resulting single-strands probably serve as templates for RNA synthesis. The multifunctional nsp13 protein additionally possesses nucleotide triphosphatase activity $[27,29,30]$ and is likely to be involved in removal of one of the terminal phosphate groups at the 5 ' end of the positivesense RNAs, which is the first step in the formation of the 5' cap structure. Although the enzyme that subsequently adds the guanine to the terminal phosphates (guanylyl transferase) has not been identified yet, nsp14 has been shown to exert S-adenosyl-L-methionine (AdoMet)-dependent (guanine-N7)methyltransferase (N7-MTase) activity [31]. Finally, the cap-1 structure is formed by the AdoMetdependent (nucleoside-2'O)-methyltransferase (2'O-MTase) activity that is present in nsp16 [32], and for which the latter needs to form a complex with nsp10 [33,34].

CoVs possess the largest genomes among the +RNA viruses [10]. They encode a large number of additional RNA-modifying enzymes, which are often not present in other RNA viruses. These additional enzymatic activities are probably required to ensure proper RNA synthesis and might account for their large size. In addition to the cap N7-MTase activity, nsp14 has metal ion-dependent 3'-to-5' exoribonuclease (ExoN) activity [35] and contains a nidoviral uridylate-specific endoribonuclease (NendoU; nsp15) [36], both able to degrade RNA and dsRNA [36,37]. While the function of the NendoU activity in the $\mathrm{CoV}$ infection cycle is not known, it appears that the ExoN activity is required to ensure high replication fidelity of the extremely large CoV genome [38,39] and that nsp14 may function as a proofreading enzyme [40].

\subsection{Nonstructural Transmembrane Proteins}

CoVs encode three nsps that contain hydrophobic stretches that are predicted to function as transmembrane domains: nsp3, nsp4 and nsp6. Consistently, membrane association has been demonstrated for the nsp3, nsp4 and nsp6 proteins of SARS-CoV [41-43] and MHV [42-45]. Both nsp3 and nsp4 become $N$-glycosylated upon insertion into the membranes of the endoplasmic reticulum (ER) [42-44]. Interestingly, transmembrane domain predictions based on the multiple alignment of $27 \mathrm{CoV}$ replicase polyprotein sequences revealed an uneven number of hydrophobic domains for both nsp3 and nsp6 [42]. This prediction is peculiar as it would separate the viral proteinases residing in $\mathrm{nsp} 3$ and nsp5 from their target sequences, implying that some of these 
hydrophobic domains might actually not span the membrane. In agreement herewith, and in contrast to the predictions, all three nsps were shown to have both their amino terminus and their carboxy terminus exposed in the cytoplasm. While all four hydrophobic domains of nsp4 span the lipid bilayer, this is the case for only two of the three hydrophobic domains in nsp3 and for six of the seven in nsp6 $[42,45]$. This experimentally established topology model (Figure 1C) makes more sense, as it positions all of the proteinase cleavage sites on the same side of the membrane as the viral proteinases themselves. Proteolytic processing of the replicase polyproteins probably starts during translation and prior to membrane insertion. Interestingly, the cleavage between nsp3 and nsp4 appears to be a rapid event $[41,46,47]$. This cleavage at the amino-terminus of the first hydrophobic/transmembrane domain of nsp4 probably facilitates the membrane insertion of nsp4 as it may enable the first hydrophobic domain to function as a signal peptide. The occurrence of conserved non-membrane spanning hydrophobic domains in nsp3 and nsp6, which are likely to be peripherally associated with the membrane, suggests an important function for these domains, possibly in the biogenesis of the $\mathrm{CoV}$ replicative structures. In this respect it is of interest to mention that the seventh hydrophobic domain of nsp6 contains putative palmitoylation sites (our own predictions and [45]). The addition of palmitic acid to this hydrophobic domain may stabilize its peripheral membrane association.

\subsection{Nonstructural Proteins with Other Functions}

In addition to the nsps mentioned above, other CoV nsps are involved in RNA binding (nsp9 and nsp10; $[48,49])$ or in evasion of the antiviral response of the host (nsp1 and nsp3; [50-57]). The function of nsp2 is not yet known, although this protein was shown not to be essential for virus replication $[58,59]$. The reader is referred to several excellent reviews on this topic for more detailed insights $[10,60,61]$.

\section{Membrane Rearrangements}

\subsection{Organelle-like Membranous Replicative Structures}

Upon infection of host cells, CoVs induce a variety of membranous structures of which some have been associated with viral RNA synthesis. The first detectable membrane rearrangements in $\mathrm{CoV}$-infected cells are 200 to $350 \mathrm{~nm}$ organelle-like structures that have been described for both MHV [47,62] and the SARS-CoV $[5,63]$ and consist of spherical vesicles containing double lipid bilayers, termed DMVs (Figure 2). In between the clusters of DMVs, reticular CMs are characteristically present $[4,62,64]$. Later in infection large virion-containing vesicles (LVCVs) [4,5,62,65], highly organized cubic membrane structures $[5,62]$ and condensed tubular bodies $[62,64]$ are formed. The latter two structures are likely a result of the overexpression of $\mathrm{CoV}$ structural proteins during infection and do not seem to be involved in CoV replication [62].

Electron tomography studies demonstrated that in SARS-CoV infected cells the DMVs and CMs form an interconnected membranous network that is also continuous with the ER [4]. This latter observation is in agreement with previous reports describing DMVs in close proximity to the ER or continuous with it $[5,47,63]$. Moreover, (partial) colocalization of replicase proteins together with the ER resident protein disulfide isomerase (PDI) has been reported [63], while also the translocon subunit 
Sec61 $\alpha$ was found to be redistributed to the replicative structures upon SARS-CoV infection [66]. The combined data indicate that the ER is the most likely membrane donor for the DMVs, despite the absence of most conventional ER markers on these structures [43,62,63,67].

Figure 2. Coronavirus-induced organelle-like replicative structures. (A) Upon coronavirus infection, replicative structures consisting of double-membrane vesicles (DMVs) and convoluted membranes (CMs) are generated; (B) A higher magnification clearly demonstrates that the DMVs contain a double-lipid bilayer. The DMVs are indicated by arrowheads and the CMs by asterisks. The size of the scale bars is indicated.
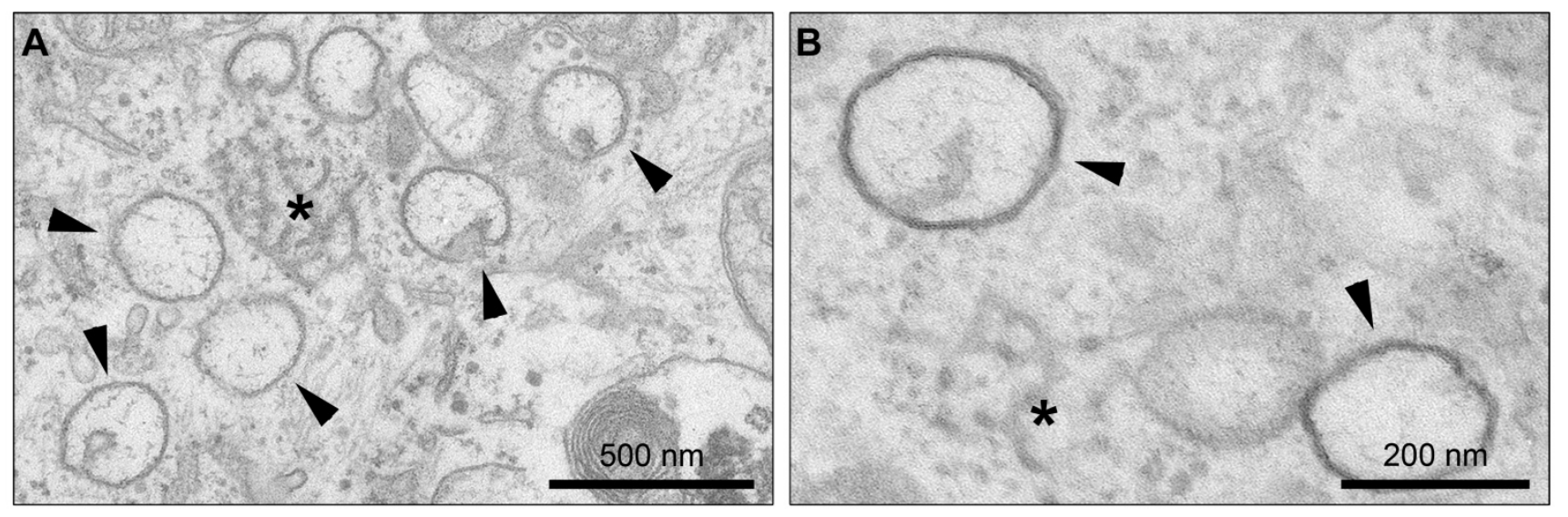

The CoV replicative structures, i.e., DMVs and CMs (Figure 2), have been associated with viral RNA synthesis, as the MHV and SARS-CoV nsps have been shown to localize to these structures [4,5,19,43,59,62,63,68-71]. In addition, antibodies recognizing dsRNA, the presumed replicative intermediates, label the interior of the SARS-CoV-induced DMVs [4]. Newly synthesized viral RNA, visualized by 5-bromouridine 5'-triphosphate (BrUTP) labeling, was observed in MHVinfected cells in close proximity to the replicative structures by immunoelectron microscopy [47,71].

\subsection{Involvement of Cellular Pathways}

Remodeling of eukaryotic cellular membranes into the replicative structures is likely dependent on the combined effects of both viral and cellular proteins and probably also on the specific lipid composition of the membranes themselves. Nevertheless, only few studies have been published addressing the involvement of cellular constituents in $\mathrm{CoV}$ replication and the generation of the replicative structures. It is conceivable that CoVs hijack cellular pathways to meet the conditions that are required for their replication, consequently adopting intrinsic properties of the utilized pathways themselves.

In agreement with the ER being the most likely membrane donor of the DMVs, an intimate association between the early secretory pathway and CoV replication has been demonstrated. RTC formation and replication in MHV-infected cells were inhibited when the secretory pathway was interfered with by blocking protein export at ER exit sites by treatment with the kinase inhibitor H89 or by expression of a dominant active Sar1 mutant [43]. Also treatment with Brefeldin A (BFA), an inhibitor of ER-to-Golgi trafficking, or knockdown of its target GBF1, inhibited MHV replication while reducing the number of DMVs [67]. Similar results were published for SARS-CoV infected cells 
treated with BFA and it was noticed that the inner and outer membranes of the DMVs were separated in BFA-treated cells [66], which may explain the observed inhibition of viral replication.

By their main ultrastructural characteristic, the double lipid bilayer, CoV DMVs very much resemble autophagosomes. This similarity prompted studies into the role of the autophagy machinery in $\mathrm{CoV}$ replication. Initial studies revealed a colocalization between the autophagosomal protein marker microtubule-associated protein light-chain 3 (LC3/Atg8) with the replicative structures [68]; moreover, viral replication was impaired and DMVs were not detected in the absence of the essential autophagy protein Atg5 [72]. In other studies however, these colocalization data could not be reproduced [63], while the absence of Atg5 did not affect CoV replication [73,74]. Yet, LC3 was found in association with the replicative structures by Zhao and coworkers [73]. Furthermore, others showed that, while the endogenous LC3 protein was recruited to the replicative structures, this was not the case for a GFP-tagged form of LC3 [69,75,76] that is often used as a marker for autophagosomes [77]. In agreement herewith, $\mathrm{CoV}$ replication was shown to be unaffected in autophagy-deficient cells lacking Atg7, although depletion of LC3 severely affected CoV replication [69]. Unlike autophagosomes, which may also be induced by expression of CoV nsp6 [74], CoV replicative structures were shown to be decorated with the non-lipidated form of LC3. Similar findings were also reported for EDEMosomes [78,79], ER-derived vesicles that transport ER chaperones to lysosomes. As the EDEMosome cargo proteins EDEM1 and OS-9 were also detected in association with the CoV replicative structures, it was proposed that CoVs hijack EDEMosomes for their replication $[69,80]$. In agreement herewith, the transmembrane SEL1L protein, which was recently shown to interact with LC3 and to have a critical function in EDEMosome biosynthesis, was also shown to colocalize with dsRNA foci in CoV-infected cells, while its depletion negatively affected CoV replication [80].

\subsection{Role of Viral Proteins}

The CoV nonstructural membrane proteins, nsp3, 4 and 6 probably play an essential role in the membrane rearrangements required for the induction of the replicative structures and in the anchoring of the RTCs to these structures. These proteins were shown to be engaged in homo- and heterotypic interactions [81]. Interestingly, co-expression of nsp4 with the C-terminal one-third part of nsp3 $\left(n s p 3_{C}\right)$ resulted in the relocalization of these proteins from the ER into discrete foci mostly localizing to the perinuclear region of the cell [81]. Although nsp6 was not required for the observed relocalization, it was recruited to the perinuclear foci when co-expressed [82], in agreement with this protein interacting with nsp4 [81]. Ultrastructural analysis of cells co-expressing nsp4 and nsp $3_{\mathrm{C}}$ revealed that the membranes of the ER exhibited more curvature, although these membranes did not resemble the DMVs observed in CoV-infected cells [82], which may not be surprising as only the Cterminal part of nsp3 was used in these co-expressions with nsp4. Similar membrane rearrangements were not observed when the nsp3 from MHV was co-expressed with nsp4 from SARS-CoV (or vice versa), while (deletion) mutagenesis studies indicated essential roles for the large luminal loops of nsp3 and nsp4 in the relocalization of these proteins [82]. While co-immunoprecipitation and immunofluorescence assays indicate that nsp3 and nsp4 of MHV interact, this interaction could not be confirmed using the Venus protein-fragment complementation assay [81]. We hypothesize that the interactions between nsp3 and nsp4 mediate some kind of "zippering" of the lipid bilayers of the ER, 
which ultimately leads to the formation of the DMVs. In this model nsp3 and nsp4 interact via their luminal loops in such a way that their interaction prevents reconstitution of a functional Venus protein.

Several other studies also suggest an important role for nsp4 in the generation of the replicative structures. Disruption of the nsp4 glycosylation sites present in the loop between the first and second hydrophobic region, leads to the formation of aberrant DMVs in which the inner and outer membranes are detached, while the number of CMs is increased [83]. In agreement herewith, although the fourth hydrophobic domain of nsp4 is dispensable, the other three transmembrane regions are required for $\mathrm{CoV}$ replication [84]. Furthermore, co-expression of the counterparts of the CoV nsp3 and nsp4 of the distantly-related equine arteritis virus (EAV), resulted in the rearrangement of host cell membranes into DMVs, albeit with a morphology [85] differing from that observed in EAV-infected cells [86]. Mutations of cysteine residues present in the luminal loop of EAV nsp3 (the EAV counterpart of CoV nsp4) resulted in altered morphologies of the DMVs, while the introduction of a $N$-glycosylation site in this loop also affected their morphology to some extent [87].

Like CoVs, other +RNA viruses also somehow induce membrane rearrangements that are required for their replication and transcription. For several of these viruses similar membrane rearrangements can be induced by the (co-)expression of nsps (for reviews see [1-3]). These nsps are either integral transmembrane proteins, examples being the NS4A proteins of Dengue virus (DENV) [88] and Kunjin virus [89] and the NS4B protein of hepatitis C virus (HCV) [90], or alternatively the proteins are only peripherally associated to the lipid bilayer, such as the 1a protein of brome mosaic virus (BMV) [91] and the $2 \mathrm{C}$ protein of poliovirus (PV) [92]. Strikingly, however, also for the integral membrane proteins the occurrence of hydrophobic/amphipathic regions that do not span the lipid bilayer but are peripherally associated with membranes, which has also been demonstrated for CoV nsp3 and nsp6 [42,44,45], appears to be a common feature [88,93-95]. Another similarity among the nsps of different + RNA viruses appears to be their ability to assemble into larger protein complexes. Such interactions have been observed not only between nsp3, nsp4 and nsp6 of CoVs, but also between the nsps of other +RNA viruses, including the BMV 1a protein [96,97] and the flavivirus NS4A and NS4B proteins [98,99].

How are the nsps of CoVs able to induce the observed membrane rearrangements? We speculate that the similarities between the membrane-associated nsps of different + RNA viruses relate to their common ability to remodel membranes. The induction of membrane curvature in lipid bilayers is critical when remodeling host cellular membranes. The membrane-associated viral proteins may act as multimeric scaffolds able to impose such curvature, for instance by acting as wedges by inserting their amphipatic helices partially into one side of the bilayer (reviewed in [100-102]). The viral proteins may function similar to host cellular proteins known to induce membrane bending via a scaffold mechanism, as exemplified by the COPI and COPII complexes, and/or via the insertion of amphipathic domains into the lipid bilayer, as has been proposed for the small GTPase Sar1 (reviewed in $[100,102])$. 


\section{Dynamics of CoV Replicative Structures and Associated Proteins}

The last few decades have provided virologists with exciting new information regarding the functions and structures of individual nsps and the characterization and formation of the membranous structures induced by +RNA viruses. These insights were obtained by classical biochemical, immunofluorescent and ultrastructural approaches. Unfortunately, little information regarding the dynamics of the (viral) proteins present at the membranous replicative structures in living cells is known. Such studies are important as classical approaches only provide static views of cellular processes and do not necessarily reflect the dynamics underlying virus replication in living cells. To date, only few studies on the dynamics of the replicative structures of plus-strand RNA viruses and their associated proteins have been published [103-109].

\subsection{Dynamics of the CoV Replicative Structures}

By using a GFP-tagged version of nsp2, shown by immuno-electron microscopy (IEM) to be recruited to the DMVs and CMs, as a marker the dynamics of the $\mathrm{CoV}$ replicative structures were studied using live cell imaging [110]. These studies showed for the first time that the CoV replicative structures are moving through the cell. However, it appeared that the CoV replicative structures consist of two classes that demonstrate different mobilities: large structures lacking any displacement and smaller structures with relatively high saltatory mobility. The smaller replicative structures were hypothesized to correspond to individual DMVs, while the larger ones supposedly represent the DMV/CM assemblies that have been observed in ultrastructural studies of CoV-infected cells [4,62]. In SARS-CoV-infected cells the DMVs are confined to a reticulovesicular network [4]. We therefore speculate that the small structures have not yet been 'captured' into this network. However, correlative light-electron microscopy studies will be required to solve this issue.

Live cell imaging studies of HCV- and Semliki Forest virus (SFV)-infected cells also reveal the presence of replicative structures that could be discriminated on the basis of their size and mobility. $\mathrm{HCV}$ induces the formation of a so-called membranous web, in which DMVs can be observed [90]. Large HCV structures, probably representing membranous webs, exhibited limited movement, whereas smaller ones were mobile and could travel long distances throughout the cytoplasm [108]. SFV-induced vacuoles are assembled at the plasma membrane after which they are transported to modified lysosomes [106,107]. SFV-infected cells also harbor large acidic immobile perinuclear vesicles and smaller acidic cytoplasmic vesicles that showed saltatory movements. In addition to these acidic vesicles, SFV-infected cells contain a class of non-acidic highly mobile vesicles that displayed multidirectional short-distance movements. Moreover, fusion of the neutral mobile structures with the acidic mobile structures resulted in the formation of the large acidic structures [107]. Such events of fusion of smaller replicative structures into larger ones have not been observed (yet) for the CoV and HCV replicative structures [108,110].

The calculated velocities of the saltatory movements of the smaller nsp2-positive structures in $\mathrm{CoV}$-infected cells [110] correspond to those measured for microtubule-mediated transport [111]. The observed association of these structures with microtubules and the inhibition of trafficking in the presence of a microtubule network-disturbing drug confirmed the transport of the smaller structures on 
microtubular tracks [110]. A role of the cytoskeleton in the movement of replicative structures has also been described for HCV, SFV, and PV [105,107-109]. Disruption of a functional microtubular network inhibited the movement of the small HCV and nascent PV replicative structures [108,109] and the trafficking of SFV neutral vesicles to acidic organelles [107], concomitant with dispersal of these structures throughout the cytoplasm. Strikingly, inhibition of microtubule-dependent trafficking did not or only modestly affect the replication of these viruses [109,110,112]. Disruption of the actin network also did not affect $\mathrm{CoV}$ replication much, although the replicative structures again failed to accumulate in the perinuclear area [113]. Collectively, these results show that the cytoskeleton is required for the perinuclear accumulation of the replicative structure rather than for replication per se. Additional studies are required to clarify the role of the perinuclear targeting of the CoV replicative structures during infection.

\subsection{Mobility of Replicative Structure-Associated Proteins}

Up till now, only few studies have addressed the dynamics of individual viral proteins when present at the replicative structures. Recently, we analyzed the dynamic properties of three replication-associated proteins, i.e., the soluble nsp2 and $\mathrm{N}$ proteins and the integral membrane protein nsp4, and demonstrated that these proteins display different diffusional mobilities when present at the replicative structures.

Nsp2, when expressed in trans is recruited to the replicative structures [110]. After recruitment, nsp2 was shown to be immobilized by using fluorescent recovery after photobleaching (FRAP) methodology. In other words, nsp2 already associated with the replicative structures was not exchanged by nsp2 present at other locations in the cell. Similar results have been demonstrated for other +RNA virus replication-associated proteins, although the published literature on this subject is limited. Also in HCV-infected cells, NS5A-positive structures showed a static internal architecture when (part of) the NS5A fluorescent protein pool was bleached [108]. When expressed individually, NS5A is highly mobile [103], similar to nsp2. Apparently, in the context of a viral infection, when other viral proteins are present, MHV nsp2 and HCV NS5A are immobilized at the replicative structures presumably due to protein-RNA or protein-protein interactions. In agreement herewith, large-scale protein-protein interaction studies demonstrated that nsp2 of MHV and SARS-CoV is engaged in a multitude of interactions with itself, nsp3, nsp4, nsp6, nsp7, nsp8, nsp11, nsp15 and nsp16 [82,114-116]. These observations are remarkable in view of the dispensability of nsp2 during $\mathrm{CoV}$ infection in vitro [59]. Yet, nsp2 has to be somehow important in vivo as its coding sequence is maintained during evolution.

In addition to its structural role in the coronavirion, i.e., in packaging of the genomic RNA into the RNP complex, the structural $\mathrm{N}$ protein is also important in coronavirus replication and has been detected in the perinuclear region of infected cells colocalizing with markers for the replicative structures $[62,67,117,118]$. The interaction of the $\mathrm{N}$ protein with nsp3 is presumably important for the initial recruitment of $\mathrm{N}$ to the replicative structures [119], while N-N protein interactions were sufficient for recruitment of (mutant) $\mathrm{N}$ proteins in the presence of wild type $\mathrm{N}$ proteins [120]. In contrast to nsp2, the $\mathrm{N}$ protein is not immobilized at the CoV replicative structures but is associated with it rather dynamically [120]. This may not be surprising, as the multifunctional $\mathrm{N}$ protein is both involved in viral replication [121-123] and virion assembly [124]. As the CoV replicative structures 
and virion assembly sites appear to be spatially separated, the newly synthesized genomic viral RNA needs to be transported from the replication sites to the assembly sites. The $\mathrm{N}$ protein presumably facilitates this transport, consistent with its dynamic behavior.

When expressed in trans in infected cells, the nsp4-GFP fusion protein was detected at the ER and at the replicative structures $[43,81]$. By performing fluorescence loss in photobleaching (FLIP) experiments continuity was demonstrated between the membranes of the ER and the replicative structures that harbor nsp4 [81], in agreement with the model that the DMVs and CMs form an interconnected network that is continuous with the ER [4]. However, nsp4 displayed different diffusional mobilities at different subcellular locations [81]. It was more mobile in the ER than at the replicative structures. This reduced mobility may be (partly) due to its engagement in interactions with the other transmembrane-containing nsps as well as with itself [81]. Also the mobility of the HCV NS4B protein, which is an integral transmembrane protein as well, depends on its intracellular location. When expressed in the absence of other viral proteins, this protein is present at the ER and at so-called membrane-associated foci (MAFs) that are induced upon expression of this protein [104]. FRAP analysis showed that NS4B present at the MAFs had a reduced mobility compared to NS4B at the ER, which was suggested to result from NS4B being engaged in different interactions when present on MAFs or the ER [104].

\section{CoV Replicative Structures and RNA Synthesis}

Currently, one of the most enigmatic issues regarding $\mathrm{CoV}$ replication is the precise localization of the sites of active viral RNA synthesis. Although CoV RNA synthesis appears to be protected by membranes [125] it is still unclear whether synthesis of nascent viral RNA occurs at sites of dsRNA accumulation, as pores connecting the interior of the coronavirus DMVs with the cytoplasm have not been detected [4], while the nsps localize to both DMVs and CMs [4,5,19,43,59,62,63,68-71]. To identify sites of nascent viral RNA synthesis, one has to define what actually constitutes the active viral replication complexes. Although dsRNA molecules function as intermediates of replication and transcription, their presence at certain sites per se does not imply (all) these structures to be actively involved in RNA synthesis. Likewise, the location of viral enzymes that are required for RNA synthesis does not need to correlate with active RTCs. Moreover, newly synthesized RNAs are not necessarily located at their site of synthesis as they may diffuse or be transported away to other subcellular locations. In view of these considerations, sites active in RNA synthesis are expected to contain at least three components: the RdRp, dsRNA intermediates active in replication/transcription and nascent viral RNA.

Newly synthesized viral RNAs, visualized by 5-bromouridine 5'-triphosphate (BrUTP) labeling, were shown to colocalize with antibodies recognizing either nsp5 or the C-terminal part of pp1a [71], and were observed in close proximity to the DMVs by immunoelectron microscopy in MHV-infected cells $[47,71]$. Recently, a new method was used to detect and visualize newly synthesized coronaviral RNA by incorporation of an alkyne-modified uridine analog, 5-ethynyl uridine (EU), onto which an azide-derivatized fluophore was coupled via a copper (I)-catalyzed cycloaddition reaction (click chemistry). With this method, it was shown that throughout MHV infection foci of nascent RNAs could be detected, which colocalize with the RdRp-containing nsp12, indicating that they correspond 
with sites of active coronaviral RNA synthesis. The relationship between nascent RNA and dsRNA is, however, less clear. While early in infection nascent RNAs colocalize at or adjacent to patches of dsRNA dots, presumably corresponding to DMVs, this correlation is much less apparent at later times when the dsRNA dots are spread throughout the cell. Many dsRNA dots are apparently not transcriptionally active as no EU labeling was associated with them, while many foci of EU labeling did not appear to colocalize with the dsRNA dots [113].

Different models can be put forward to explain these observations. In one model, DMVs function as the sites of active RNA synthesis. At the later times in infection, many DMVs are no longer active, while the ones that are active may contain only little dsRNA, resulting in less apparent colocalization of dsRNA and EU labeling. In another model, DMVs are non-functional end-stage products. They are not actively involved in RNA synthesis, but rather harbor dsRNAs that are not (longer) functioning as intermediates in RNA synthesis. In this model, which is in agreement with the presumed absence of pores in these structures [4], the CMs would be the only plausible alternative for the sites of active RNA synthesis. In yet another model, DMVs may be the initial sites of active RNA synthesis, particularly early in infection, while at later times the membranes become sealed, connections are lost and RNA synthesis shifts to the CM assemblies. Clearly, ultrastructural studies will be required, ideally (co)localizing nascent RNAs as well as the RdRp, to definitely determine the precise localization of CoV RNA synthesis.

For most other +RNA viruses the identification of the sites of active RNA synthesis appears less complicated. Nascent RNAs, as well as nsps, have been shown to label the spherules that are observed in BMV- [126], SFV- [106] or flock house virus (FHV)- [127] infected cells at the modified ER, lysosomes and mitochondria, respectively, indicating that these structures correspond with the sites of active RNA synthesis. Nascent RNAs were previously also shown to colocalize with dsRNA in Kunjin flavivirus-infected cells [128]. Electron tomography of the membrane rearrangements observed in flavirus-infected cells revealed that the inner content of the DMVs, which contains nsps and dsRNA, is connected to the cytoplasm via a pore $[129,130]$, indicating that these DMVs are actually spherule-like invaginations (once) active in RNA synthesis.

\section{Future Directions}

$\mathrm{CoV}$ replicative structures/RTCs are macromolecular assemblies, the components of which are engaged in a plethora of protein-protein, protein-RNA, and protein-lipid interactions [114-116,131]. Currently, the exact composition of the replicative structures/RTCs is not known, let alone the full arsenal of interactions occurring within these structures. Moreover, the replicative structures are likely to be subject to some form of maturation [14,19,20,113], as their composition appears to change during the course of infection as determined by biochemical and immunofluorescence analyses. Thus, it will be of interest to confirm, extend and refine the previously published protein-protein interactome studies that have been published for SARS-CoV [114-116], for example by investigating proteinprotein interactions of other CoVs using novel (large-scale) screening approaches. Likewise, it will be of interest to get more insight into the involvement of host proteins in the formation of these structures, for example by screening for host proteins that interact with the CoV nsps or by elucidating the protein content of purified replicative structures -preferably in time- using mass spectrometry. 
One of the difficulties associated with these studies is the complexity of discriminating whether host proteins are directly involved in the formation of the replicative structures themselves or in RNA synthesis per se, as inhibition of either process will result in reduced RNA synthesis, protein expression and DMV formation. Therefore, assays are needed in which the formation of replicative structures can be studied independent of viral replication. Such assays may be provided by co-expression of viral proteins $[81,85]$ that induce the rearrangement of cellular membranes. Recently, by using such a replication-independent assay, reticulons, which form a family of ER membraneshaping proteins, were implicated in the formation of the BMV-induced, ER-derived spherules that are associated with viral RNA synthesis [132]. Reticulons are involved in the induction of ER membrane curvature [133-135] and seem to be required by BMV for determining the size of the spherules and for stabilizing the spherule necks [132]. It will be of interest to study the putative role of these proteins in $\mathrm{CoV}$ replication and in reshaping of the ER membranes by $\mathrm{CoV}$ nsps. Other proteins playing a role in shaping and remodeling of the ER are the atlastins and CLIMP-63 [133,136]. Also these proteins are putative candidates involved in $\mathrm{CoV}$-induced membrane remodeling and would deserve to be investigated.

In addition to the role of virus and host proteins in formation of the replicative structures, lipids may also be important in this process. Dynamic alteration of the lipid composition can induce curvature of membranes to some extent $[100,102,137,138]$, which is, however, unlikely to be sufficient to generate the organelle-like structures observed in +RNA virus-infected cells. It is more likely that lipids, together with lipid-modifying enzymes, contribute to the formation of the replicative structures by providing a suitable microenvironment to which the viral (and cellular) membrane shaping proteins are recruited. Even more, +RNA viruses may specifically hijack lipid-modifying enzymes for their own advantage $[139,140]$. This is underscored by several studies, which show that inhibition of lipid synthesis by using either drugs or small interfering RNAs dramatically affects the replication of +RNA viruses [139,141-144]. It will be of interest to study how and to what extent CoV infection affects the lipid homeostasis in infected cells, for example by analyzing the lipid repertoire of infected cells by lipidomics techniques.

Finally, as all processes occurring in living cells are inherently dynamic in nature, it is also desirable to get more insight into the biogenesis and functioning of the replicative structures using various live cell imaging approaches. For example, photoactivatable fluorescent proteins can be used to investigate the formation of the replicative structures in real-time by 'optical pulse-labeling' in living cells, while the behavior of individual nsps associated with the RTCs can be studied by selectively 'switching-on' (sub)populations of proteins [145,146]. Furthermore, tagging of viral RNA by genetic incorporation of specific RNA sequences that bind fluorescently-tagged RNA-binding proteins [147,148], hybridization of fluorescent 'molecular beacons' to the viral RNAs [149], or combining metabolic labeling of viral RNAs with $\mathrm{Cu}$-independent click chemistry [150], will allow visualization and tracking of these ribonucleic acid species in living cells. Concomitant live cell imaging of viral RNA and the N proteins may be used to investigate the transport of nascent RNA from the replication to the assembly sites. In addition, super resolution microscopy techniques, like photoactivated localization microscopy (PALM), stimulated emission depletion microscsopy (STED) and stochastic optical reconstruction microscopy (STORM) [151-153] can all be applied to investigate the $\mathrm{CoV}$ RTCs and the membranous replicative structures at the ultrastructural level using fluorescently-tagged proteins, while EU-labeling of viral RNA in combination with correlative light- 
electron microscopy may provide the resolution to indisputably pinpoint the exact location of viral RNA synthesis [154].

\section{Acknowledgments}

We would like to thank Oliver Wicht and Christine Burkhard from the Virology Division, Faculty of Veterinary Medicine, Utrecht University for stimulating discussion and Fulvio Reggiori and Mustafa Ulasli from the Department of Cell Biology and Cell Microscopy Center, University Medical Centre Utrecht for providing the EM pictures of the $\mathrm{CoV}$ replicative structures. C.A.M.D.H. is supported by grants from the Netherlands Organization for Scientific Research (NWO-ALW) and Utrecht University (High Potential).

\section{Conflict of Interest}

The authors declare no conflict of interest.

\section{References and Notes}

1. den Boon, J.A.; Ahlquist, P. Organelle-Like Membrane Compartmentalization of Positive-Strand RNA Virus Replication Factories. Annu. Rev. Microbiol. 2010, 64, 241-256.

2. den Boon, J.A.; Diaz, A.; Ahlquist, P. Cytoplasmic Viral Replication Complexes. Cell Host Microbe 2010, 8, 77-85.

3. Miller, S.; Krijnse-Locker, J. Modification of Intracellular Membrane Structures for Virus Replication. Nat. Rev. Microbiol. 2008, 6, 363-74.

4. Knoops, K.; Kikkert, M.; van den Worm, S.H.E.; Zevenhoven-Dobbe, J.C.; van der Meer, Y.; Koster, A.J.; Mommaas, A.M.; Snijder, E.J. SARS-Coronavirus Replication is Supported by a Reticulovesicular Network of Modified Endoplasmic Reticulum. PLoS Biol. 2008, 6, 1957-1974.

5. Goldsmith, C.S.; Tatti, K.M.; Ksiazek, T.G.; Rollin, P.E.; Comer, J.A.; Lee, W.W.; Rota, P.A.; Bankamp, B.; Bellini, W.J.; Zaki, S.R. Ultrastructural Characterization of SARS Coronavirus. Emerg. Infect. Dis. 2004, 10, 320-326.

6. Drosten, C.; Günther, S.; Preiser, W.; van der Werf, S.; Brodt, H.R.; Becker, S.; Rabenau, H.; Panning, M.; Kolesnikova, L.; Fouchier, R.A.M.; et al. Identification of a Novel Coronavirus in Patients with Severe Acute Respiratory Syndrome. N. Engl. J. Med. 2003, 348, 1967-1976.

7. Ksiazek, T.G.; Erdman, D.; Goldsmith, C.S.; Zaki, S.R.; Peret, T.; Emery, S.; Tong, S.; Urbani, C.; Comer, J.A.; Lim, W.; et al. A Novel Coronavirus Associated with Severe Acute Respiratory Syndrome. N. Engl. J. Med. 2003, 348, 1953-1966.

8. Guan, Y.; Zheng, B.J.; He, Y.Q.; Liu, X.L.; Zhuang, Z.X.; Cheung, C.L.; Luo, S.W.; Li, P.H.; Zhang, L.J.; Guan, Y.J.; et al. Isolation and Characterization of Viruses Related to the SARS Coronavirus from Animals in Southern China. Science 2003, 302, 276-278.

9. Li, W.; Shi, Z.; Yu, M.; Ren, W.; Smith, C.; Epstein, J.H.; Wang, H.; Crameri, G.; Hu, Z.; Zhang, H.; et al. Bats are Natural Reservoirs of SARS-Like Coronaviruses. Science 2005, 310, $676-679$. 
10. Gorbalenya, A.E.; Enjuanes, L.; Ziebuhr, J.; Snijder, E.J. Nidovirales: Evolving the Largest RNA Virus Genome. Virus Res. 2006, 117, 17-37.

11. Sawicki, S.G.; Sawicki, D.L.; Siddell, S.G. A Contemporary View of Coronavirus Transcription. J. Virol. 2007, 81, 20-29.

12. Sawicki, S.G.; Sawicki, D.L. Coronaviruses use Discontinuous Extension for Synthesis of Subgenome-Length Negative Strands. Adv. Exp. Med. Biol. 1995, 380, 499-506.

13. Zuniga, S.; Sola, I.; Alonso, S.; Enjuanes, L. Sequence Motifs Involved in the Regulation of Discontinuous Coronavirus Subgenomic RNA synthesis. J. Virol. 2004, 78, 980-994.

14. Sawicki, S.G.; Sawicki, D.L. Coronavirus Minus-Strand RNA Synthesis and Effect of Cycloheximide on Coronavirus RNA Synthesis. J. Virol. 1986, 57, 328-334.

15. Sawicki, D.; Wang, T.; Sawicki, S. The RNA Structures Engaged in Replication and Transcription of the A59 Strain of Mouse Hepatitis Virus. J. Gen. Virol. 2001, 82, 385-396.

16. Bredenbeek, P.J.; Pachuk, C.J.; Noten, A.F.; Charite, J.; Luytjes, W.; Weiss, S.R.; Spaan, W.J. The Primary Structure and Expression of the Second Open Reading Frame of the Polymerase Gene of the Coronavirus MHV-A59; a Highly Conserved Polymerase is Expressed by an Efficient Ribosomal Frameshifting Mechanism. Nucleic Acids Res. 1990, 18, 1825-1832.

17. Brierley, I.; Digard, P.; Inglis, S.C. Characterization of an Efficient Coronavirus Ribosomal Frameshifting Signal: Requirement for an RNA Pseudoknot. Cell 1989, 57, 537-547.

18. Ziebuhr, J.; Snijder, E.J.; Gorbalenya, A.E. Virus-Encoded Proteinases and Proteolytic Processing in the Nidovirales. J. Gen. Virol. 2000, 81, 853-879.

19. Deming, D.J.; Graham, R.L.; Denison, M.R.; Baric, R.S. Processing of Open Reading Frame 1a Replicase Proteins nsp7 to nsp10 in Murine Hepatitis Virus Strain A59 Replication. J. Virol. 2007, 81, 10280-10291.

20. Sawicki, S.G.; Sawicki, D.L.; Younker, D.; Meyer, Y.; Thiel, V.; Stokes, H.; Siddell, S.G. Functional and Genetic Analysis of Coronavirus Replicase-Transcriptase Proteins. PLoS Pathog. 2005, 1 , e39.

21. te Velthuis, A.J.; Arnold, J.J.; Cameron, C.E.; van den Worm, S.H.; Snijder, E.J. The RNA Polymerase Activity of SARS-Coronavirus nsp12 is Primer Dependent. Nucleic Acids Res. 2010, $38,203-214$.

22. Imbert, I.; Guillemot, J.C.; Bourhis, J.M.; Bussetta, C.; Coutard, B.; Egloff, M.P.; Ferron, F.; Gorbalenya, A.E.; Canard, B. A Second, Non-Canonical RNA-Dependent RNA Polymerase in SARS Coronavirus. EMBO J. 2006, 25, 4933-4942.

23. Zhai, Y.; Sun, F.; Li, X.; Pang, H.; Xu, X.; Bartlam, M.; Rao, Z. Insights into SARS-CoV Transcription and Replication from the Structure of the nsp7-nsp8 Hexadecamer. Nat. Struct. Mol. Biol. 2005, 12, 980-986.

24. Xiao, Y.; Ma, Q.; Restle, T.; Shang, W.; Svergun, D.I.; Ponnusamy, R.; Sczakiel, G.; Hilgenfeld, R. Nonstructural Proteins 7 and 8 of Feline Coronavirus Form a 2:1 Heterotrimer that Exhibits Primer-Independent RNA Polymerase Activity. J. Virol. 2012, 86, 4444-4454.

25. te Velthuis, A.J.; van den Worm, S.H.; Snijder, E.J. The SARS-Coronavirus nsp7+nsp8 Complex is a Unique Multimeric RNA Polymerase Capable of both De Novo Initiation and Primer Extension. Nucleic Acids Res. 2012, 40, 1737-1747. 
26. Seybert, A.; Posthuma, C.C.; van Dinten, L.C.; Snijder, E.J.; Gorbalenya, A.E.; Ziebuhr, J. A Complex Zinc Finger Controls the Enzymatic Activities of Nidovirus Helicases. J. Virol. 2005, 79, 696-704.

27. Seybert, A.; Hegyi, A.; Siddell, S.G.; Ziebuhr, J. The Human Coronavirus 229E Superfamily 1 Helicase has RNA and DNA Duplex-Unwinding Activities with 5'-to-3' Polarity. RNA 2000, 6, 1056-1068.

28. Tanner, J.A.; Watt, R.M.; Chai, Y.B.; Lu, L.Y.; Lin, M.C.; Peiris, J.S.; Poon, L.L.; Kung, H.F.; Huang, J.D. The Severe Acute Respiratory Syndrome (SARS) Coronavirus NTPase/helicase Belongs to a Distinct Class of 5' to 3' Viral Helicases. J. Biol. Chem. 2003, 278, 39578-39582.

29. Ivanov, K.A.; Thiel, V.; Dobbe, J.C.; van der Meer, Y.; Snijder, E.J.; Ziebuhr, J. Multiple Enzymatic Activities Associated with Severe Acute Respiratory Syndrome Coronavirus Helicase. J. Virol. 2004, 78, 5619-5632.

30. Ivanov, K.A.; Ziebuhr, J. Human Coronavirus 229E Nonstructural Protein 13: Characterization of Duplex-Unwinding, Nucleoside Triphosphatase, and RNA 5'-Triphosphatase Activities. J. Virol. 2004, 78, 7833-7838.

31. Chen, Y.; Cai, H.; Pan, J.; Xiang, N.; Tien, P.; Ahola, T.; Guo, D. Functional Screen Reveals SARS Coronavirus Nonstructural Protein nsp14 as a Novel Cap N7 Methyltransferase. Proc. Natl. Acad. Sci. USA 2009, 106, 3484-3489.

32. Decroly, E.; Imbert, I.; Coutard, B.; Bouvet, M.; Selisko, B.; Alvarez, K.; Gorbalenya, A.E.; Snijder, E.J.; Canard, B. Coronavirus Nonstructural Protein 16 is a Cap-0 Binding Enzyme Possessing (Nucleoside-2'O)-Methyltransferase Activity. J. Virol. 2008, 82, 8071-8084.

33. Bouvet, M.; Debarnot, C.; Imbert, I.; Selisko, B.; Snijder, E.J.; Canard, B.; Decroly, E. In Vitro Reconstitution of SARS-Coronavirus mRNA Cap Methylation. PLoS Pathog. 2010, 6, e1000863.

34. Lugari, A.; Betzi, S.; Decroly, E.; Bonnaud, E.; Hermant, A.; Guillemot, J.C.; Debarnot, C.; Borg, J.P.; Bouvet, M.; Canard, B. et al. Molecular Mapping of the RNA Cap 2'-O-Methyltransferase Activation Interface between Severe Acute Respiratory Syndrome Coronavirus nsp10 and nsp16. J. Biol. Chem. 2010, 285, 33230-33241.

35. Minskaia, E.; Hertzig, T.; Gorbalenya, A.E.; Campanacci, V.; Cambillau, C.; Canard, B.; Ziebuhr, J. Discovery of an RNA Virus $3 ' \rightarrow 5$ ' Exoribonuclease that is Critically Involved in Coronavirus RNA Synthesis. Proc. Natl. Acad. Sci. USA 2006, 103, 5108-5113.

36. Ivanov, K.A.; Hertzig, T.; Rozanov, M.; Bayer, S.; Thiel, V.; Gorbalenya, A.E.; Ziebuhr, J. Major Genetic Marker of Nidoviruses Encodes a Replicative Endoribonuclease. Proc. Natl. Acad. Sci. USA 2004, 101, 12694-12699.

37. Bhardwaj, K.; Sun, J.; Holzenburg, A.; Guarino, L.A.; Kao, C.C. RNA Recognition and Cleavage by the SARS Coronavirus Endoribonuclease. J. Mol. Biol. 2006, 361, 243-256.

38. Eckerle, L.D.; Becker, M.M.; Halpin, R.A.; Li, K.; Venter, E.; Lu, X.; Scherbakova, S.; Graham, R.L.; Baric, R.S.; Stockwell, T.B.; et al. Infidelity of SARS-CoV Nsp14-Exonuclease Mutant Virus Replication is Revealed by Complete Genome Sequencing. PLoS Pathog. 2010, 6, e1000896.

39. Denison, M.R.; Graham, R.L.; Donaldson, E.F.; Eckerle, L.D.; Baric, R.S. Coronaviruses: An RNA Proofreading Machine Regulates Replication Fidelity and Diversity. RNA Biol. 2011, 8 , $270-279$. 
40. Bouvet, M.; Imbert, I.; Subissi, L.; Gluais, L.; Canard, B.; Decroly, E. RNA 3'-End Mismatch Excision by the Severe Acute Respiratory Syndrome Coronavirus Nonstructural Protein nsp10/nsp14 Exoribonuclease Complex. Proc. Natl. Acad. Sci. USA 2012, 109, 9372-9377.

41. Harcourt, B.H.; Jukneliene, D.; Kanjanahaluethai, A.; Bechill, J.; Severson, K.M.; Smith, C.M.; Rota, P.A.; Baker, S.C. Identification of Severe Acute Respiratory Syndrome Coronavirus Replicase Products and Characterization of Papain-Like Protease Activity. J. Virol. 2004, 78, 13600-13612.

42. Oostra, M.; Hagemeijer, M.C.; van Gent, M.; Bekker, C.P.; te Lintelo, E.G.; Rottier, P.J.; de Haan, C.A. Topology and Membrane Anchoring of the Coronavirus Replication Complex: Not all Hydrophobic Domains of nsp3 and nsp6 are Membrane Spanning. J. Virol. 2008, 82, 12392-12405.

43. Oostra, M.; te Lintelo, E.G.; Deijs, M.; Verheije, M.H.; Rottier, P.J.; de Haan, C.A. Localization and Membrane Topology of Coronavirus Nonstructural Protein 4: Involvement of the Early Secretory Pathway in Replication. J. Virol. 2007, 81, 12323-12336.

44. Kanjanahaluethai, A.; Chen, Z.; Jukneliene, D.; Baker, S.C. Membrane Topology of Murine Coronavirus Replicase Nonstructural Protein 3. Virology 2007, 361, 391-401.

45. Baliji, S.; Cammer, S.A.; Sobral, B.; Baker, S.C. Detection of Nonstructural Protein 6 in Murine Coronavirus-Infected Cells and Analysis of the Transmembrane Topology by using Bioinformatics and Molecular Approaches. J. Virol. 2009, 83, 6957-6962.

46. Kanjanahaluethai, A.; Baker, S.C. Identification of Mouse Hepatitis Virus Papain-like Proteinase 2 Activity. J. Virol. 2000. 74, 7911-7921.

47. Gosert, R.; Kanjanahaluethai, A.; Egger, D.; Bienz, K.; Baker, S.C. RNA Replication of Mouse Hepatitis Virus Takes Place at Double-Membrane Vesicles. J. Virol. 2002, 76, 3697-3708.

48. Sutton, G.; Fry, E.; Carter, L.; Sainsbury, S.; Walter, T.; Nettleship, J.; Berrow, N.; Owens, R.; Gilbert, R.; Davidson, A.; et al. The nsp9 Replicase Protein of SARS-Coronavirus, Structure and Functional Insights. Structure 2004, 12, 341-353.

49. Su, D.; Lou, Z.; Sun, F.; Zhai, Y.; Yang, H.; Zhang, R.; Joachimiak, A.; Zhang, X.C.; Bartlam, M.; Rao, Z. Dodecamer Structure of Severe Acute Respiratory Syndrome Coronavirus Nonstructural Protein nsp10. J. Virol. 2006, 80, 7902-7908.

50. Zust, R.; Cervantes-Barragan, L.; Kuri, T.; Blakqori, G.; Weber, F.; Ludewig, B.; Thiel, V. Coronavirus Non-Structural Protein 1 is a Major Pathogenicity Factor: Implications for the Rational Design of Coronavirus Vaccines. PLoS Pathog. 2007, 3, e109.

51. Wang, G.; Chen, G.; Zheng, D.; Cheng, G.; Tang, H. PLP2 of Mouse Hepatitis Virus A59 (MHV-A59) Targets TBK1 to Negatively Regulate Cellular Type I Interferon Signaling Pathway. PLoS One 2011, 6, e17192.

52. Wathelet, M.G.; Orr, M.; Frieman, M.B.; Baric, R.S. Severe Acute Respiratory Syndrome Coronavirus Evades Antiviral Signaling: Role of nsp1 and Rational Design of an Attenuated Strain. J. Virol. 2007, 81, 11620-11633.

53. Narayanan, K.; Huang, C.; Lokugamage, K.; Kamitani, W.; Ikegami, T.; Tseng, C.T.; Makino, S. Severe Acute Respiratory Syndrome Coronavirus nsp1 Suppresses Host Gene Expression, Including that of Type I Interferon, in Infected Cells. J. Virol. 2008, 82, 4471-4479.

54. Kuri, T.; Eriksson, K.K.; Putics, A.; Zust, R.; Snijder, E.J.; Davidson, A.D.; Siddell, S.G.; Thiel, V.; Ziebuhr, J.; Weber, F. The ADP-Ribose-1"-Monophosphatase Domains of SARS-Coronavirus 
and Human Coronavirus 229E Mediate Resistance to Antiviral Interferon Responses. J. Gen. Virol. 2011.

55. Kamitani, W.; Narayanan, K.; Huang, C.; Lokugamage, K.; Ikegami, T.; Ito, N.; Kubo, H.; Makino, S. Severe Acute Respiratory Syndrome Coronavirus nsp1 Protein Suppresses Host Gene Expression by Promoting Host mRNA Degradation. Proc. Natl. Acad. Sci. USA 2006, 103, 12885-12890.

56. Kamitani, W.; Huang, C.; Narayanan, K.; Lokugamage, K.G.; Makino, S. A Two-Pronged Strategy to Suppress Host Protein Synthesis by SARS Coronavirus Nsp1 Protein. Nat. Struct. Mol. Biol. 2009, 16, 1134-1140.

57. Chen, C.J.; Sugiyama, K.; Kubo, H.; Huang, C.; Makino, S. Murine Coronavirus Nonstructural Protein p28 Arrests Cell Cycle in G0/G1 Phase. J. Virol. 2004, 78, 10410-10419.

58. Gadlage, M.J.; Graham, R.L.; Denison, M.R. Murine Coronaviruses Encoding nsp2 at Different Genomic Loci have Altered Replication, Protein Expression, and Localization. J. Virol. 2008, 82, 11964-11969.

59. Graham, R.L.; Sims, A.C.; Brockway, S.M.; Baric, R.S.; Denison, M.R. The nsp2 Replicase Proteins of Murine Hepatitis Virus and Severe Acute Respiratory Syndrome Coronavirus are Dispensable for Viral Replication. J. Virol. 2005, 79, 13399-13411.

60. Perlman, S.; Netland, J. Coronaviruses Post-SARS: Update on Replication and Pathogenesis. Nat. Rev. Microbiol. 2009, 7, 439-450.

61. Ziebuhr, J. The Coronavirus Replicase: Insights into a Sophisticated Enzyme Machinery. Adv. Exp. Med. Biol. 2006, 581, 3-11.

62. Ulasli, M.; Verheije, M.H.; de Haan, C.A.; Reggiori, F. Qualitative and Quantitative Ultrastructural Analysis of the Membrane Rearrangements Induced by Coronavirus. Cell. Microbiol. 2010, 12, 844-861.

63. Snijder, E.J.; van der Meer, Y.; Zevenhoven-Dobbe, J.; Onderwater, J.J.; van der Meulen, J.; Koerten, H.K.; Mommaas, A.M. Ultrastructure and Origin of Membrane Vesicles Associated with the Severe Acute Respiratory Syndrome Coronavirus Replication Complex. J. Virol. 2006, 80, 5927-5940.

64. David-Ferreira, J.F.; Manaker, R.A. An Electron Microscope Study of the Development of a Mouse Hepatitis Virus in Tissue Culture Cells. J. Cell. Biol. 1965, 24, 57-78.

65. Ng, M.L.; Tan, S.H.; See, E.E.; Ooi, E.E.; Ling, A.E. Proliferative Growth of SARS Coronavirus in Vero E6 Cells. J. Gen. Virol. 2003, 84, 3291-3303.

66. Knoops, K.; Swett-Tapia, C.; van den Worm, S.H.E.; te Velthuis, A.J.W.; Koster, A.J.; Mommaas, A.M.; Snijder, E.J.; Kikkert, M. Integrity of the Early Secretory Pathway Promotes, but is Not Required for, Severe Acute Respiratory Syndrome Coronavirus RNA Synthesis and Virus-Induced Remodeling of Endoplasmic Reticulum Membranes. J. Virol. 2010, 84, 833-846.

67. Verheije, M.H.; Raaben, M.; Mari, M.; Te Lintelo, E.G.; Reggiori, F.; van Kuppeveld, F.J.; Rottier, P.J.; de Haan, C.A. Mouse Hepatitis Coronavirus RNA Replication Depends on GBF1Mediated ARF1 Activation. PLoS Pathog. 2008, 4, e1000088.

68. Prentice, E.; McAuliffe, J.; Lu, X.; Subbarao, K.; Denison, M.R. Identification and Characterization of Severe Acute Respiratory Syndrome Coronavirus Replicase Proteins. J. Virol. 2004, 78, 9977-9986. 
69. Reggiori, F.; Monastyrska, I.; Verheije, M.H.; Calì, T.; Ulasli, M.; Bianchi, S.; Bernasconi, R.; de Haan, C.A.M.; Molinari, M. Coronaviruses Hijack the LC3-I-Positive EDEMosomes, ER-Derived Vesicles Exporting Short-Lived ERAD Regulators, for Replication. Cell Host Microbe 2010, 7, 500-508.

70. Shi, S.T.; Schiller, J.J.; Kanjanahaluethai, A.; Baker, S.C.; Oh, J.W.; Lai, M.M. Colocalization and Membrane Association of Murine Hepatitis Virus Gene 1 Products and De Novo-Synthesized Viral RNA in Infected Cells. J. Virol. 1999, 73, 5957-5969.

71. van der Meer, Y.; Snijder, E.J.; Dobbe, J.C.; Schleich, S.; Denison, M.R.; Spaan, W.J.; Locker, J.K. Localization of Mouse Hepatitis Virus Nonstructural Proteins and RNA Synthesis Indicates a Role for Late Endosomes in Viral Replication. J. Virol. 1999, 73, 7641-7657.

72. Prentice, E.; Jerome, W.G.; Yoshimori, T.; Mizushima, N.; Denison, M.R. Coronavirus Replication Complex Formation Utilizes Components of Cellular Autophagy. J. Biol. Chem. 2004, 279, 10136-10141.

73. Zhao, Z.; Thackray, L.B.; Miller, B.C.; Lynn, T.M.; Becker, M.M.; Ward, E.; Mizushima, N.N.; Denison, M.R.; Virgin, H.W.,4th. Coronavirus Replication does Not Require the Autophagy Gene ATG5. Autophagy 2007, 3, 581-585.

74. Cottam, E.M.; Maier, H.J.; Manifava, M.; Vaux, L.C.; Chandra-Schoenfelder, P.; Gerner, W.; Britton, P.; Ktistakis, N.T.; Wileman, T. Coronavirus nsp6 Proteins Generate Autophagosomes from the Endoplasmic Reticulum via an Omegasome Intermediate. Autophagy 2011, 7, 1335-1347.

75. de Haan, C.A.M.; Reggiori, F.; Molinari, M. Autophagy-Independent LC3 Function in Vesicular Traffic. Autophagy 2010, 6, 994-996.

76. Stertz, S.; Reichelt, M.; Spiegel, M.; Kuri, T.; Martinez-Sobrido, L.; Garcia-Sastre, A.; Weber, F.; Kochs, G. The Intracellular Sites of Early Replication and Budding of SARS-Coronavirus. Virology 2007, 361, 304-315.

77. Mizushima, N.; Yamamoto, A.; Matsui, M.; Yoshimori, T.; Ohsumi, Y. In Vivo Analysis of Autophagy in Response to Nutrient Starvation using Transgenic Mice Expressing a Fluorescent Autophagosome Marker. Mol. Biol. Cell 2004, 15, 1101-1111.

78. Cali, T.; Galli, C.; Olivari, S.; Molinari, M. Segregation and Rapid Turnover of EDEM1 by an Autophagy-Like Mechanism Modulates Standard ERAD and Folding Activities. Biochem. Biophys. Res. Commun. 2008, 371, 405-410.

79. Cali, T.; Vanoni, O.; Molinari, M. The Endoplasmic Reticulum Crossroads for Newly Synthesized Polypeptide Chains. Prog. Mol. Biol. Transl. Sci. 2008, 83, 135-179.

80. Bernasconi, R.; Galli, C.; Noack, J.; Bianchi, S.; de Haan, C.A.; Reggiori, F.; Molinari, M. Role of the SEL1L:LC3-I Complex as an ERAD Tuning Receptor in the Mammalian ER. Mol. Cell. 2012, 46, 809-19.

81. Hagemeijer, M.C.; Ulasli, M.; Vonk, A.; Reggiori, F.; Rottier, P.J.M.; de Haan, C.A.M. Mobility and Interactions of the Coronavirus Nonstructural Protein 4. J. Virol. 2011, 85, 4572-7.

82. Hagemeijer, M.C.; de Haan, C.A.M. Utrecht University, Utrecht, the Netherlands, Unpublished work, 2012. 
83. Gadlage, M.J.; Sparks, J.S.; Beachboard, D.C.; Cox, R.G.; Doyle, J.D.; Stobart, C.C.; Denison, M.R. Murine Hepatitis Virus Nonstructural Protein 4 Regulates Virus-Induced Membrane Modifications and Replication Complex Function. J. Virol. 2010, 84, 280-290.

84. Sparks, J.S.; Lu, X.; Denison, M.R. Genetic Analysis of Murine Hepatitis Virus nsp4 in Virus Replication. J. Virol. 2007, 81, 12554-63.

85. Snijder, E.J.; van Tol, H.; Roos, N.; Pedersen, K.W. Non-Structural Proteins 2 and 3 Interact to Modify Host Cell Membranes during the Formation of the Arterivirus Replication Complex. J. Gen. Virol. 2001, 82, 985-994.

86. Pedersen, K.W.; van der Meer, Y.; Roos, N.; Snijder, E.J. Open Reading Frame 1a-Encoded Subunits of the Arterivirus Replicase Induce Endoplasmic Reticulum-Derived Double-Membrane Vesicles which Carry the Viral Replication Complex. J. Virol. 1999, 73, 2016-2026.

87. Posthuma, C.C.; Pedersen, K.W.; Lu, Z.; Joosten, R.G.; Roos, N.; Zevenhoven-Dobbe, J.C.; Snijder, E.J. Formation of the Arterivirus replication/transcription Complex: A Key Role for Nonstructural Protein 3 in the Remodeling of Intracellular Membranes. J. Virol. 2008, 82, 4480-4491.

88. Miller, S.; Kastner, S.; Krijnse-Locker, J.; Buhler, S.; Bartenschlager, R. The Non-Structural Protein 4A of Dengue Virus is an Integral Membrane Protein Inducing Membrane Alterations in a 2K-Regulated Manner. J. Biol. Chem. 2007, 282, 8873-8882.

89. Roosendaal, J.; Westaway, E.G.; Khromykh, A.; Mackenzie, J.M. Regulated Cleavages at the West Nile Virus NS4A-2K-NS4B Junctions Play a Major Role in Rearranging Cytoplasmic Membranes and Golgi Trafficking of the NS4A Protein. J. Virol. 2006, 80, 4623-4632.

90. Egger, D.; Wolk, B.; Gosert, R.; Bianchi, L.; Blum, H.E.; Moradpour, D.; Bienz, K. Expression of Hepatitis C Virus Proteins Induces Distinct Membrane Alterations Including a Candidate Viral Replication Complex. J. Virol. 2002, 76, 5974-5984.

91. den Boon, J.A.; Chen, J.; Ahlquist, P. Identification of Sequences in Brome Mosaic Virus Replicase Protein 1a that Mediate Association with Endoplasmic Reticulum Membranes. J. Virol. 2001, 75, 12370-12381.

92. Echeverri, A.C.; Dasgupta, A. Amino Terminal Regions of Poliovirus 2C Protein Mediate Membrane Binding. Virology 1995, 208, 540-553.

93. Lundin, M.; Monne, M.; Widell, A.; von Heijne, G.; Persson, M.A.A. Topology of the Membrane-Associated Hepatitis C Virus Protein NS4B. J. Virol. 2003, 77, 5428-5438.

94. Miller, D.J.; Ahlquist, P. Flock House Virus RNA Polymerase is a Transmembrane Protein with Amino-Terminal Sequences Sufficient for Mitochondrial Localization and Membrane Insertion. J. Virol. 2002, 76, 9856-9867.

95. Miller, S.; Sparacio, S.; Bartenschlager, R. Subcellular Localization and Membrane Topology of the Dengue Virus Type 2 Non-Structural Protein 4B. J. Biol. Chem. 2006, 281, 8854-8863.

96. O'Reilly, E.K.; Wang, Z.; French, R.; Kao, C.C. Interactions between the Structural Domains of the RNA Replication Proteins of Plant-Infecting RNA Viruses. J. Virol. 1998, 72, 7160-7169.

97. O'Reilly, E.; Paul, J.; Kao, C. Analysis of the Interaction of Viral RNA Replication Proteins by using the Yeast Two-Hybrid Assay. J. Virol. 1997, 71, 7526-7532.

98. Paul, D.; Romero-Brey, I.; Gouttenoire, J.; Stoitsova, S.; Krijnse-Locker, J.; Moradpour, D.; Bartenschlager, R. NS4B Self-Interaction through Conserved C-Terminal Elements is Required 
for the Establishment of Functional Hepatitis C Virus Replication Complexes. J. Virol. 2011, 85, 6963-6976.

99. Mackenzie, J.M.; Khromykh, A.A.; Jones, M.K.; Westaway, E.G. Subcellular Localization and some Biochemical Properties of the Flavivirus Kunjin Nonstructural Proteins NS2A and NS4A. Virology 1998, 245, 203-215.

100. McMahon, H.T.; Gallop, J.L. Membrane Curvature and Mechanisms of Dynamic Cell Membrane Remodelling. Nature 2005, 438, 590-596.

101. Shibata, Y.; Hu, J.; Kozlov, M.M.; Rapoport, T.A. Mechanisms Shaping the Membranes of Cellular Organelles. Annu. Rev. Cell Dev. Biol. 2009, 25, 329-354.

102.Zimmerberg, J.; Kozlov, M.M. How Proteins Produce Cellular Membrane Curvature. Nat. Rev. Mol. Cell Biol. 2006, 7, 9-19.

103. Jones, D.M.; Gretton, S.N.; McLauchlan, J.; Targett-Adams, P. Mobility Analysis of an NS5A-GFP Fusion Protein in Cells Actively Replicating Hepatitis C Virus Subgenomic RNA. J. Gen. Virol. 2007, 88, 470-475.

104. Gretton, S.N.; Taylor, A.I.; McLauchlan, J. Mobility of the Hepatitis C Virus NS4B Protein on the Endoplasmic Reticulum Membrane and Membrane-Associated Foci. J. Gen. Virol. 2005, 86, $1415-1421$.

105. Cui, Z.; Zhang, Z.; Zhang, X.; Wen, J.; Zhou, Y.; Xie, W. Visualizing the Dynamic Behavior of Poliovirus Plus-Strand RNA in Living Host Cells. Nucleic Acids Res. 2005, 33, 3245-3252.

106. Kujala, P.; Ikaheimonen, A.; Ehsani, N.; Vihinen, H.; Auvinen, P.; Kaariainen, L. Biogenesis of the Semliki Forest Virus RNA Replication Complex. J. Virol. 2001, 75, 3873-3884.

107. Spuul, P.; Balistreri, G.; Kaariainen, L.; Ahola, T. Phosphatidylinositol 3-Kinase-, Actin-, and Microtubule-Dependent Transport of Semliki Forest Virus Replication Complexes from the Plasma Membrane to Modified Lysosomes. J. Virol. 2010, 84, 7543-7557.

108. Wolk, B.; Buchele, B.; Moradpour, D.; Rice, C.M. A Dynamic View of Hepatitis C Virus Replication Complexes. J. Virol. 2008, 82, 10519-10531.

109. Egger, D.; Bienz, K. Intracellular Location and Translocation of Silent and Active Poliovirus Replication Complexes. J. Gen. Virol. 2005, 86, 707-718.

110. Hagemeijer, M.C.; Verheije, M.H.; Ulasli, M.; Shaltiël, I.A.; de Vries, L.A.; Reggiori, F.; Rottier, P.J.M.; de Haan, C.A.M. Dynamics of Coronavirus Replication-Transcription Complexes. J. Virol. 2010, 84, 2134-2149.

111. Ma, S.; Chisholm, R.L. Cytoplasmic Dynein-Associated Structures Move Bidirectionally in Vivo. J. Cell Sci. 2002, 115, 1453-1460.

112. Bost, A.G.; Venable, D.; Liu, L.; Heinz, B.A. Cytoskeletal Requirements for Hepatitis C Virus (HCV) RNA Synthesis in the HCV Replicon Cell Culture System. J. Virol. 2003, 77, 4401-4408.

113. Hagemeijer, M.C.; Vonk, A.M.; Monastyrska, I.; Rottier, P.J.; de Haan, C.A. Visualizing Coronavirus RNA Synthesis in Time by using Click Chemistry. J. Virol. 2012, 86, 5808-5816.

114. Imbert, I.; Snijder, E.J.; Dimitrova, M.; Guillemot, J.C.; Lecine, P.; Canard, B. The SARSCoronavirus PLnc Domain of nsp3 as a replication/transcription Scaffolding Protein. Virus Res. 2008, 133, 136-148. 
115. Pan, J.; Peng, X.; Gao, Y.; Li, Z.; Lu, X.; Chen, Y.; Ishaq, M.; Liu, D.; DeDiego, M.L.; Enjuanes, L.; et al. Genome-Wide Analysis of Protein-Protein Interactions and Involvement of Viral Proteins in SARS-CoV Replication. PLoS One 2008, 3, e3299.

116. von Brunn, A.; Teepe, C.; Simpson, J.C.; Pepperkok, R.; Friedel, C.C.; Zimmer, R.; Roberts, R.; Baric, R.; Haas, J. Analysis of Intraviral Protein-Protein Interactions of the SARS Coronavirus ORFeome. PLoS One 2007, 2, e459.

117. Bost, A.G.; Carnahan, R.H.; Lu, X.T.; Denison, M.R. Four Proteins Processed from the Replicase Gene Polyprotein of Mouse Hepatitis Virus Colocalize in the Cell Periphery and Adjacent to Sites of Virion Assembly. J. Virol. 2000, 74, 3379-3387.

118. Denison, M.R.; Spaan, W.J.; van der Meer, Y.; Gibson, C.A.; Sims, A.C.; Prentice, E.; Lu, X.T. The Putative Helicase of the Coronavirus Mouse Hepatitis Virus is Processed from the Replicase Gene Polyprotein and Localizes in Complexes that are Active in Viral RNA Synthesis. J. Virol. 1999, 73, 6862-6871.

119. Hurst, K.R.; Ye, R.; Goebel, S.J., Javaraman, P.; Masters, P.S. An Interaction between the Nucleocapsid Protein and a Component of the Replicase-Transcriptase Complex is Crucial for the Infectivity of Coronavirus Genomic RNA. J. Virol. 2010, 84, 10276-10288.

120. Verheije, M.H.; Hagemeijer, M.C.; Ulasli, M.; Reggiori, F.; Rottier, P.J.M.; Masters, P.S.; de Haan, C.A.M. The Coronavirus Nucleocapsid Protein is Dynamically Associated with the Replication-Transcription Complexes. J. Virol. 2010, 84, 11575-9.

121. Almazan, F.; Galan, C.; Enjuanes, L. The Nucleoprotein is Required for Efficient Coronavirus Genome Replication. J. Virol. 2004, 78, 12683-12688.

122. Compton, S.R.; Rogers, D.B.; Holmes, K.V.; Fertsch, D.; Remenick, J.; McGowan, J.J. In Vitro Replication of Mouse Hepatitis Virus Strain A59. J. Virol. 1987, 61, 1814-1820.

123. Schelle, B.; Karl, N.; Ludewig, B.; Siddell, S.G.; Thiel, V. Selective Replication of Coronavirus Genomes that Express Nucleocapsid Protein. J. Virol. 2005, 79, 6620-6630.

124. de Haan, C.A.; Rottier, P.J. Molecular Interactions in the Assembly of Coronaviruses. Adv. Virus Res. 2005, 64, 165-230.

125. van Hemert, M.J.; van den Worm, S.H.; Knoops, K.; Mommaas, A.M.; Gorbalenya, A.E.; Snijder, E.J. SARS-Coronavirus replication/transcription Complexes are Membrane-Protected and Need a Host Factor for Activity in Vitro. PLoS Pathog. 2008, 4, e1000054.

126. Schwartz, M.; Chen, J.; Janda, M.; Sullivan, M.; den Boon, J.; Ahlquist, P. A Positive-Strand RNA Virus Replication Complex Parallels Form and Function of Retrovirus Capsids. Mol. Cell 2002, 9, 505-514.

127. Kopek, B.G.; Perkins, G.; Miller, D.J.; Ellisman, M.H.; Ahlquist, P. Three-Dimensional Analysis of a Viral RNA Replication Complex Reveals a Virus-Induced Mini-Organelle. PLoS Biol. 2007, 5 , e220.

128. Westaway, E.G.; Khromykh, A.A.; Mackenzie, J.M. Nascent Flavivirus RNA Colocalizedin Situwith Double-Stranded RNA in Stable Replication Complexes. Virology 1999, 258, $108-117$.

129. Gillespie, L.K.; Hoenen, A.; Morgan, G.; Mackenzie, J.M. The Endoplasmic Reticulum Provides the Membrane Platform for Biogenesis of the Flavivirus Replication Complex. J. Virol. 2010, 84, $10438-10447$. 
130. Welsch, S.; Miller, S.; Romero-Brey, I.; Merz, A.; Bleck, C.K.; Walther, P.; Fuller, S.D.; Antony, C.; Krijnse-Locker, J.; Bartenschlager, R. Composition and Three-Dimensional Architecture of the Dengue Virus Replication and Assembly Sites. Cell Host Microbe 2009, 5, 365-375.

131. Sola, I.; Mateos-Gomez, P.A.; Almazan, F.; Zuniga, S.; Enjuanes, L. RNA-RNA and RNA-Protein Interactions in Coronavirus Replication and Transcription. RNA Biol. 2011, 8, 237-248.

132. Diaz, A.; Wang, X.; Ahlquist, P. Membrane-Shaping Host Reticulon Proteins Play Crucial Roles in Viral RNA Replication Compartment Formation and Function. Proc. Natl. Acad. Sci. USA 2010, 107, 16291-16296.

133. Hu, J.; Shibata, Y.; Zhu, P.P.; Voss, C.; Rismanchi, N.; Prinz, W.A.; Rapoport, T.A.; Blackstone, C. A Class of Dynamin-Like GTPases Involved in the Generation of the Tubular ER Network. Cell 2009, 138, 549-561.

134. Hu, J.; Shibata, Y.; Voss, C.; Shemesh, T.; Li, Z.; Coughlin, M.; Kozlov, M.M.; Rapoport, T.A.; Prinz, W.A. Membrane Proteins of the Endoplasmic Reticulum Induce High-Curvature Tubules. Science 2008, 319, 1247-1250.

135. Voeltz, G.K.; Prinz, W.A.; Shibata, Y.; Rist, J.M.; Rapoport, T.A. A Class of Membrane Proteins Shaping the Tubular Endoplasmic Reticulum. Cell 2006, 124, 573-586.

136. Klopfenstein, D.R.; Klumperman, J.; Lustig, A.; Kammerer, R.A.; Oorschot, V.; Hauri, H. Subdomain-Specific Localization of Climp-63 (P63) in the Endoplasmic Reticulum is Mediated by its Luminal $\alpha$-Helical Segment. J. Cell Biol. 2001, 153, 1287-1300.

137. Farsad, K.; Camilli, P.D. Mechanisms of Membrane Deformation. Curr. Opin. Cell Biol. 2003, 15, 372-381.

138. Shibata, Y.; Shemesh, T.; Prinz, W.A.; Palazzo, A.F.; Kozlov, M.M.; Rapoport, T.A. Mechanisms Determining the Morphology of the Peripheral ER. Cell 2010, 143, 774-788.

139. Hsu, N.; Ilnytska, O.; Belov, G.; Santiana, M.; Chen, Y.; Takvorian, P.M.; Pau, C.; van der Schaar, H.; Kaushik-Basu, N.; Balla, T.; et al. Viral Reorganization of the Secretory Pathway Generates Distinct Organelles for RNA Replication. Cell 2010, 141, 799-811.

140. Reiss, S.; Rebhan, I.; Backes, P.; Romero-Brey, I.; Erfle, H.; Matula, P.; Kaderali, L.; Poenisch, M.; Blankenburg, H.; Hiet, M.; et al. Recruitment and Activation of a Lipid Kinase by Hepatitis C Virus NS5A is Essential for Integrity of the Membranous Replication Compartment. Cell Host Microbe 2011, 9, 32-45.

141. Guinea, R.; Carrasco, L. Phospholipid Biosynthesis and Poliovirus Genome Replication, Two Coupled Phenomena. EMBO J. 1990, 9, 2011-2016.

141. Heaton, N.S.; Perera, R.; Berger, K.L.; Khadka, S.; LaCount, D.J.; Kuhn, R.J.; Randall, G. Dengue Virus Nonstructural Protein 3 Redistributes Fatty Acid Synthase to Sites of Viral Replication and Increases Cellular Fatty Acid Synthesis. Proc. Natl. Acad. Sci. USA 2010, 107, 17345-17350.

143. Perez, L.; Guinea, R.; Carrasco, L. Synthesis of Semliki Forest Virus RNA Requires Continuous Lipid Synthesis. Virology 1991, 183, 74-82.

144. Perera, R.; Riley, C.; Isaac, G.; Hopf-Jannasch, A.S.; Moore, R.J.; Weitz, K.W.; Pasa-Tolic, L.; Metz, T.O.; Adamec, J.; Kuhn, R.J. Dengue Virus Infection Perturbs Lipid Homeostasis in Infected Mosquito Cells. PLoS Pathog. 2012, 8, e1002584. 
145. Lippincott-Schwartz, J.; Altan-Bonnet, N.; Patterson, G.H. Photobleaching and Photoactivation: Following Protein Dynamics in Living Cells. Nat. Cell. Biol. 2003, Suppl., S7-S14.

146. Lippincott-Schwartz, J.; Patterson, G.H. Development and use of Fluorescent Protein Markers in Living Cells. Science 2003, 300, 87-91.

147. Fusco, D.; Accornero, N.; Lavoie, B.; Shenoy, S.M.; Blanchard, J.; Singer, R.H.; Bertrand, E. Single mRNA Molecules Demonstrate Probabilistic Movement in Living Mammalian Cells. Curr. Biol. 2003, 13, 161-167.

148. Daigle, N.; Ellenberg, J. LambdaN-GFP: An RNA Reporter System for Live-Cell Imaging. Nat. Methods 2007, 4, 633-636.

149. Tyagi, S.; Kramer, F.R. Molecular Beacons: Probes that Fluoresce upon Hybridization. Nat. Biotechnol. 1996, 14, 303-308.

150. Baskin, J.M.; Prescher, J.A.; Laughlin, S.T.; Agard, N.J.; Chang, P.V.; Miller, I.A.; Lo, A.; Codelli, J.A.; Bertozzi, C.R. Copper-Free Click Chemistry for Dynamic in Vivo Imaging. Proc. Natl. Acad. Sci. USA 2007, 104, 16793-16797.

151. Betzig, E.; Patterson, G.H.; Sougrat, R.; Lindwasser, O.W.; Olenych, S.; Bonifacino, J.S.; Davidson, M.W.; Lippincott-Schwartz, J.; Hess, H.F. Imaging Intracellular Fluorescent Proteins at Nanometer Resolution. Science 2006, 313, 1642-1645.

152. Rust, M.J.; Bates, M.; Zhuang, X. Sub-Diffraction-Limit Imaging by Stochastic Optical Reconstruction Microscopy (STORM). Nat. Methods 2006, 3, 793-795.

153. Hell, S.W.; Wichmann, J. Breaking the Diffraction Resolution Limit by Stimulated Emission: Stimulated-Emission-Depletion Fluorescence Microscopy. Opt. Lett. 1994, 19, 780-782.

154. van Rijnsoever, C.; Oorschot, V.; Klumperman, J. Correlative Light-Electron Microscopy (CLEM) Combining Live-Cell Imaging and Immunolabeling of Ultrathin Cryosections. Nat. Methods 2008, 5, 973-980.

(C) 2012 by the authors; licensee MDPI, Basel, Switzerland. This article is an open access article distributed under the terms and conditions of the Creative Commons Attribution license (http://creativecommons.org/licenses/by/3.0/). 